\title{
THE FORMATION OF STELLAR CLUSTERS: MASS SPECTRA FROM TURBULENT MOLECULAR CLOUD FRAGMENTATION
}

\author{
RALF S. KLESSEN \\ UCO/Lick Observatory, University of California at Santa Cruz, Santa Cruz, CA 95064, USA (e-mail: ralf@ucolick.org) \\ Max-Planck-Institut für Astronomie, Königstuhl 17, 69117 Heidelberg, Germany
}

(Accepted for publication in ApJ)

\begin{abstract}
Star formation is intimately linked to the dynamical evolution of molecular clouds. Turbulent fragmentation determines where and when protostellar cores form, and how they contract and grow in mass via competitive accretion from the surrounding cloud material. This process is investigated, using numerical models of self-gravitating molecular cloud dynamics, where no turbulent support is included, where turbulence is allowed to decay freely, and where it is continuously replenished on large, intermediate and small scales, respectively. Molecular cloud regions without turbulent driving sources, or where turbulence is driven on large scales, exhibit rapid and efficient star formation in a clustered mode, whereas interstellar turbulence that carries most energy on small scales results in isolated star formation with low efficiency.

The clump mass spectrum of shock-generated density fluctuations in pure hydrodynamic, supersonic turbulence is not well fit by a power law, and it is too steep at the high-mass end to be in agreement with the observational data. When gravity is included in the turbulence models, local collapse occurs, and the spectrum extends towards larger masses as clumps merge together, a power-law description $d N / d M \propto M^{\nu}$ becomes possible with slope $\nu \lesssim-2$. In the case of pure gravitational contraction, i.e. in regions without turbulent support, the clump mass spectrum is shallower with $\nu \approx-3 / 2$.

The mass spectrum of protostellar cores in regions without turbulent support and where turbulence is replenished on large-scales, however, is well described by a log-normal or by multiple power laws, similar to the stellar IMF at low and intermediate masses. The model clusters are not massive enough to allow for comparison with the high-mass part of the IMF. In the case of small-scale turbulence, the core mass spectrum is too flat compared to the IMF for all masses.

Subject headings: hydrodynamics - ISM: clouds - ISM: kinematics and dynamics - ISM: clump mass spectrum - stars: formation - stars: IMF - turbulence
\end{abstract}

\section{INTRODUCTION}

Understanding the processes that lead to the formation of stars is one of the fundamental challenges in astronomy. As mass is the dominant parameter determining stellar evolution, reproducing and explaining the initial mass function of stars (IMF) is a key requisite for any realistic theory of star formation.

Stars are born in turbulent interstellar clouds of molecular hydrogen. The location and the mass growth of young stars are hereby intimately coupled to the dynamical cloud environment. Stars form by gravitational collapse of shock-compressed density fluctuations generated from the supersonic turbulence ubiquitously observed in molecular clouds (e.g. Elmegreen 1993, Padoan 1995, Klessen, Heitsch, \& Mac Low 2000, Padoan et al. 2001). Once a gas clump becomes gravitationally unstable, it begins to collapse and the central density increases considerably, giving birth to a protostar. In this dynamic picture, star formation takes place roughly on a free-fall timescale, as opposed to the "standard" model of the inside-out collapse of singular isothermal spheres, where core formation is dominated by the ambipolar diffusion timescale (Shu 1977, Shu, Adams, \& Lizano 1987). Altogether, star formation can be seen as a two-phase process: First, turbulent fragmentation leads to transient clumpy molecular cloud structure, with some of the density fluctuation exceeding the critical mass and density for gravitational contraction. Second, the collapse of individual Jeansunstable protostellar clumps builds up the stars. In this phase, a nascent protostar grows in mass via accretion from the infalling envelope until the available gas reservoir is exhausted or stellar feedback effects become important and remove the parental cocoon - a new star is born (e.g. André, Ward-Thompson, \& Barsony 2000, Myers, Evans, \& Ohashi 2000). The terms shockgenerated density fluctuations and gas clumps are used synonymously, and clumps are identified using a 3-dimensional clumpfinding algorithm comparable to the one described in Williams, De Geus, \& Blitz (1994). Protostellar cores in the simulations are defined as the (unresolved) high-density central regions of collapsing clumps, where individual protostars build up.

Stars form in small aggregates or larger clusters (Lada 1992, Mizuno et al. 1995, Testi, Palla, \& Natta 1998, also Adams \& Myers 2001), where the interaction of protostellar cores and their competition for mass from their surrounding may become important for shaping the distribution of the final star properties (Bonnell et al. 1997, Klessen, Burkert, \& Bate 1998, and Klessen \& Burkert 2000, 2001, in the following papers I and II, respectively). This complex evolutionary sequence involves a wide variety of different physical phenomena, and it is not at all well understood which processes dominate and determine the stellar mass spectrum.

The current investigation is the fourth in a series which focuses on the first phase of the star formation process, modeling the turbulent fragmentation of large subvolumes inside molecular clouds and the dynamical evolution towards the formation of clusters of protostellar cores. Numerical simulations (\$2) of self-gravitating isothermal gas, without turbulent support, with decaying turbulence, and with supersonic compressible turbulence that is driven on large, intermediate, and small scales, are 
TABLE 1

Properties of the Considered Molecular Cloud Models

\begin{tabular}{crccccl}
\hline \hline model & type & $k^{\mathrm{a}}$ & $\mathcal{M}_{\mathrm{rms}} \mathrm{b}^{\mathrm{b}}$ & $N_{\mathrm{J}}^{\mathrm{c}}$ & particles & further reference $^{\mathrm{d}}$ \\
\hline 1 & Gaussian density & - & - & 220 & $5 \times 10^{5}$ & model $\mathcal{I}$ in KB \\
2 & decaying turbulence & {$[1 \ldots 8]$} & {$[5.0]$} & 220 & $2 \times 10^{5}$ & - \\
3 & driven turbulence & $1 \ldots 2$ & 3.3 & 120 & $2 \times 10^{5}$ & model $\mathcal{A} 1 h$ in KHM \\
4 & driven turbulence & $3 \ldots 4$ & 3.3 & 120 & $2 \times 10^{5}$ & model $\mathcal{A} 2 h$ in KHM \\
5 & driven turbulence & $7 \ldots 8$ & 3.3 & 120 & $2 \times 10^{5}$ & model $\mathcal{A} 3 h$ in KHM \\
\hline
\end{tabular}

a Driving wavenumber (in model 2, driving stopped when gravity is 'turned on' at the stage of fully established turbulence)

${ }^{\mathrm{b}}$ Mach number in turbulent equilibrium as calculated from the 1-dimensional rms velocity dispersion, $\mathcal{M}_{\mathrm{rms}} \equiv \sigma_{1 \mathrm{D}} / c_{\mathrm{s}}$, where $c_{\mathrm{s}}$ is the sound speed and $\sigma_{1 \mathrm{D}}$ is defined via $\sigma_{1 \mathrm{D}}^{2}=1 / 3 \sigma_{3 \mathrm{D}}^{2}=1 / 3\left(E_{\mathrm{kin}} / 2\right)$ with $E_{\mathrm{kin}}$ being the total kinetic energy. Recall that the total mass is unity. For model 2 the value corresponds to time $t<0$ before the driving mechanism was 'turned off' and turbulence was allowed to decay.

${ }^{c}$ Number of (spherical) mean thermal Jeans masses contained in the system; note that this number is lower by 2 when using a cubic definition of the Jeans mass

${ }^{\mathrm{d}}$ Corresponding model name in KB (paper I) and in KHM (Klessen et al. 2000) for further details

used to analyze the star formation resulting from the interplay between gravity on the one side and gas pressure and turbulent motions on the other $(\S 3)$. In particular, the relation between the masses of molecular clumps, protostellar cores and the final stars in the considered models are discussed $(\S 4)$ and the results summarized (§5).

\section{NUMERICAL METHOD AND DRIVEN TURBULENCE}

To adequately describe turbulent fragmentation and the formation of protostellar cores, it is necessary to resolve the collapse of shock-compressed regions over several orders of magnitude in density. Due to the stochastic nature of supersonic turbulence, it is not known in advance where and when local collapse occurs. Hence, SPH (smoothed particle hydrodynamics) is used to solve the equations of hydrodynamics. It is a Lagrangian method, where the fluid is represented by an ensemble of particles and flow quantities are obtained by averaging over an appropriate subset of the SPH particles (Benz 1990). The method is able to resolve large density contrasts as particles are free to move and so naturally the particle concentration increases in high-density regions. SPH can also be combined with the special-purpose hardware device GRAPE (Sugimoto et al. 1990, Ebisuzaki et al. 1993; also Steinmetz 1996) permitting calculations at supercomputer level on a normal workstation. The simulations presented here concentrate on subregions within a much larger cloud, therefore periodic boundary conditions are adopted (Klessen 1997). Once the high-density, protostellar cores in the centers of collapsing gas clumps exceed a density limit four orders of magnitude above the mean density, they are substituted by 'sink' particles (Bate, Bonnell, \& Price 1995). These particles have the ability to accrete gas from their envelopes, while keeping track of mass and linear and angular momentum. By adequately replacing high-density core with sink particles one is able to follow the dynamical evolution of the system over many free-fall times.

The large observed linewidths in molecular clouds imply the presence of supersonic velocity fields that carry enough energy to counterbalance gravity on global scales (Williams, Blitz, \& McKee 2000). However, it is known that turbulent energy dissipates rapidly, i.e. roughly on the free-fall timescale (Mac Low et al. 1998, Stone, Ostriker, \& Gammie 1998, Padoan \& Nordlund 1999). To prevent or considerably postpone global collapse, turbulence is required to be continuously replenished. This is achieved here by applying a non-local driving scheme, that inserts energy in a limited range of wavenumbers such that the total kinetic energy contained in the system remains constant and compensates the gravitational contraction on global scales (Mac Low 1999). The models do not include magnetic fields, as their presence cannot halt the decay of turbulence (Mac Low et al. 1998, Stone et al. 1998, Padoan \& Nordlund 1999) and does not significantly alter the efficiency of local collapse for driven turbulence (Heitsch, Mac Low, \& Klessen 2001). Furthermore, possible feedback effects from the star formation process itself (like bipolar outflows, stellar winds, or ionizing radiation from new-born $\mathrm{O}$ or B stars) are neglected. This necessarily limits the interpretation of the mass spectra at very late evolutionary stages of the system. Hence, the current analysis restrains itself to phases when the mass accumulated protostellar cores is less than $\sim 70 \%$ of the total mass in the considered volume.

Altogether, five different models of molecular cloud dynamics are considered here: To compare with the case of driven turbulence, model 1 describes the dynamical evolution of an initially Gaussian density fluctuation field where turbulence is assumed to have already decayed in the considered molecular cloud region. This describes the most extreme case of clustered star formation, and the simulation is identical to model $\mathcal{I}$ in paper I. The power spectrum of the initial density fluctuations is $P(k) \propto k^{-2}$. Model 2 starts with a fully established supersonically turbulent velocity field, but turbulence is allowed to decay freely. Driven turbulence is represented by model 3 where the energy source acts at wavenumbers $k$ in the range $1 \leq k \leq 2$, by model 4 which has $3 \leq k \leq 4$, and model 5 with $7 \leq k \leq 8$. The wavelengths of the corresponding perturbations are $\ell=L / k$, where $L$ is the total size of the computed volume. Hence, ki- 
netic energy is continuously added on large, intermediate, and small scales, respectively. The driving strength is adjusted to yield the same constant turbulent Mach number $\mathcal{M}_{\text {rms }}=3.3$ for all three models (see Klessen et al. 2000, models $\mathcal{A} 1 h, \mathcal{A} 2 h$, and $\mathcal{A} 3 h$ ). Turbulence that is driven on large scales appears to yield most appropriate description of molecular cloud dynamics and star formation as is suggested by statistical analysis of molecular cloud structure (e.g. Ossenkopf \& Mac Low 2001). The relevant model parameters are listed in table 1 .

The models presented here are computed in normalized units. If scaled to mean densities of $n\left(\mathrm{H}_{2}\right)=10^{5} \mathrm{~cm}^{-3}$, a value typical for star-forming molecular cloud regions (e.g. in $\rho$-Ophiuchus, see Motte, André, \& Neri 1998) and a temperature of $11.4 \mathrm{~K}$ (i.e. a sound speed $c_{\mathrm{s}}=0.2 \mathrm{~km} \mathrm{~s}^{-1}$ ), the total mass contained in the computed volume in models 1 and 2 is $220 \mathrm{M}_{\odot}$ and the size of the cube is $0.34 \mathrm{pc}$. This corresponds to 220 thermal Jeans masses. Models 3 to 5 have a mass of $120 \mathrm{M}_{\odot}$ within a volume of $(0.29 \mathrm{pc})^{3}$, equivalent to 120 thermal Jeans masses ${ }^{1}$. In the adopted scaling, the mean thermal Jeans mass in all models is thus $\left\langle M_{\mathrm{J}}\right\rangle=1 \mathrm{M}_{\odot}$, the global free-fall timescale is $\tau_{\mathrm{ff}}=10^{5} \mathrm{yr}$, and the simulations cover a density range from $n\left(\mathrm{H}_{2}\right) \approx 100 \mathrm{~cm}^{-3}$ in the lowest density regions to $n\left(\mathrm{H}_{2}\right) \approx 10^{9} \mathrm{~cm}^{-3}$ where collapsing protostellar cores are identified and converted into 'sink' particles in the code. In this density regime gas cools very efficiently and it is possible to use an effective polytropic equation-of-state in the simulations instead of solving the detailed radiation transfer equations. The effective polytropic index is typically close to unity, $\gamma_{\text {eff }} \lesssim 1$, except for densities $10^{5} \mathrm{~cm}^{-3}<n\left(\mathrm{H}_{2}\right)<10^{7} \mathrm{~cm}^{-3}$, where smaller values of $\gamma_{\text {eff }}$ are expected (Spaans \& Silk 2000). For simplicity, a value of $\gamma_{\text {eff }}=1$, i.e. an isothermal equation of state, is adopted for all densities in the simulations. Concerning the gas temperature, this approximation is certainly valid as in star forming clouds the temperature cannot drop significantly below the adopted canonical value of $10 \mathrm{~K}$, even in the regime $10^{5} \mathrm{~cm}^{-3}<$ $n\left(\mathrm{H}_{2}\right)<10^{7} \mathrm{~cm}^{-3}$. However, it needs to be noted that the stiffness of the equation of state also determines the density contrast in shock compressed gas, and hence influences the overall density distribution in supersonic flows. For further discussions see Scalo et al. (1998), Ballesteros-Paredes, Vázquez-Semadeni, \& Scalo (1999), and Spaans \& Silk (2000). Variations in $\gamma_{\text {eff influ- }}$ ence the local Jeans scale in shock-compressed density fluctuations and may modify the resulting mass spectrum of collapsing cores. This effect needs to be investigated in more detail.

\section{STAR FORMATION FROM TURBULENT FRAGMENTATION}

Stars form from turbulent fragmentation of molecular cloud material. Supersonic turbulence, even if it strong enough to counterbalance gravity on global scales, will usually provoke local collapse. Turbulence establishes a complex network of interacting shocks, where converging shock fronts generate clumps of high density. This density enhancement can be large enough for the fluctuations to become gravitationally unstable and collapse. This happens when the local Jeans length becomes smaller than the size of the fluctuation. However, the fluctuations in turbulent velocity fields are highly transient. The random flow that creates local density enhancements can disperse them again. For local collapse to actually result in the formation of stars, Jeans-unstable shock-generated density fluctua- tions must collapse to sufficiently high densities on time scales shorter than the typical time interval between two successive shock passages. Only then are they able to 'decouple' from the ambient flow and survive subsequent shock interactions. The shorter the time between shock passages, the less likely these fluctuations are to survive. Hence, the timescale and efficiency of protostellar core formation depend strongly on the wavelength and strength of the driving source (Klessen et al. 2000, Heitsch et al. 2001), and accretion histories of individual protostars are strongly time varying (Klessen 2001, hereafter paper III).

The velocity field of long-wavelength turbulence is found to be dominated by large-scale shocks which are very efficient in sweeping up molecular cloud material, thus creating massive coherent structures. When a coherent region reaches the critical density for gravitational collapse its mass typically exceeds the local Jeans limit by far. Inside the shock compressed region, the velocity dispersion is much smaller than in the ambient turbulent flow and the situation is similar to localized turbulent decay. Quickly a cluster of protostellar cores forms. Therefore, models 1 to 3 with zero support, decaying and large-scale turbulence, respectively, lead to a clustered mode of star formation. The efficiency of turbulent fragmentation is reduced if the driving wavelength decreases. When energy is inserted mainly on small spatial scales, the network of interacting shocks is very tightly knit, and protostellar cores form independently of each other at random locations throughout the cloud and at random times. Individual shock generated clumps have lower mass and the time interval between two shock passages through the same point in space is small. Hence, collapsing cores are easily destroyed again and star formation is inefficient. This scenario corresponds to the isolated mode of star formation.

This is visualized in figure 1, showing the density structure of all five models at $t=0$ and at a time when the first protostellar cores have formed by turbulent fragmentation and have accreted roughly $30 \%$ of the total mass. For model 1 (Gaussian), without turbulent support, the figure indicates at $t=0$ the initial density distribution. For the other models, $t=0$ corresponds to the phase of fully developed turbulence just before gravity is 'switched on' (in model 2 the driving mechanism is 'switched off' at the same time). Time is measured in units of the global free-fall timescale $\tau_{\mathrm{ff}}=(3 \pi / 32 G)^{-1 / 2}\langle\rho\rangle^{-1 / 2}$, with $\langle\rho\rangle$ being the mean density and $G$ the gravitational constant. Dark dots indicate the location of dense collapsed core. In the non-supported model 1 all spatial modes are unstable initially and in model 2 of decaying turbulence they become unstable after roughly one crossing time. Therefore, these systems evolve into a filamentary structure and protostellar cores form predominantly at the intersections of the filaments. Similarly, also large-scale turbulence builds up a network of filaments, however, this time the large coherent structures are not caused by gravity, but instead are due to shock compression. Once gravity is included, it quickly dominates the evolution inside the shock compressed regions. The random velocity component is quickly damped by dissipation, and again a cluster of protostellar cores builds up. In the case of intermediate-wavelength turbulence, cores form in small aggregates, and small-scale turbulence predominantly results in the formation of isolated cores. Note the different times needed for mass to be accumulated in dense cores.

\footnotetext{
${ }^{1}$ Throughout this paper the spherical definition of the Jeans mass is used, $M_{\mathrm{J}} \equiv 4 / 3 \pi \rho \lambda_{\mathrm{J}}^{3}$, with density $\rho$ and Jeans length $\lambda_{\mathrm{J}} \equiv\left(\frac{\pi \mathcal{R} T}{G \rho}\right)^{1 / 2}$, where $G$ and $\mathcal{R}$ are the gravitational and the gas constant. The mean Jeans mass $\left\langle M_{\mathrm{J}}\right\rangle$ is then determined from average density in the system $\langle\rho\rangle$. An alternative cubic definition, $M_{\mathrm{J}} \equiv \rho\left(2 \lambda_{\mathrm{J}}\right)^{3}$, would yield a value roughly twice as large.
} 
MODEL 1
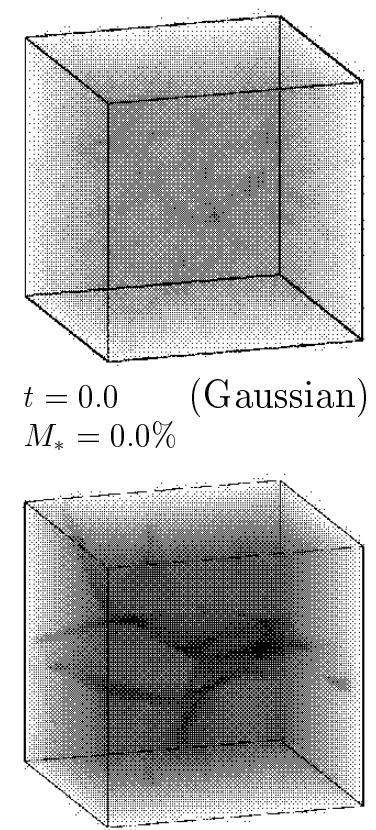

$$
t=1.1 \quad \text { (Gaussian) }
$$$$
M_{*}=28.6 \%
$$

\section{MODEL 2}
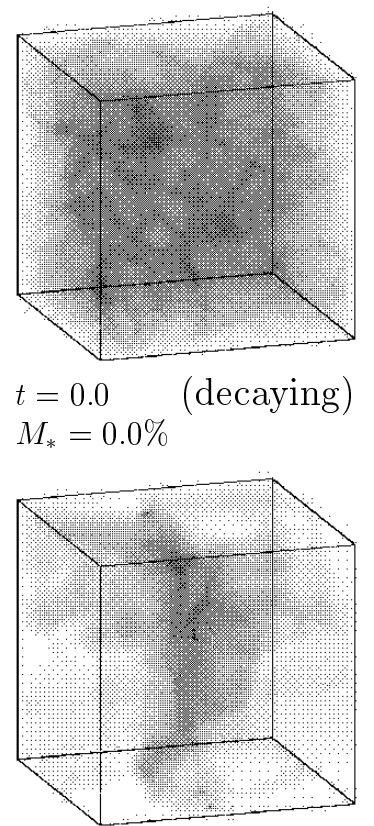

$$
\begin{aligned}
& t=1.3 \quad \text { (decaying) } \\
& M_{*}=29.3 \%
\end{aligned}
$$

\section{MODEL 3}

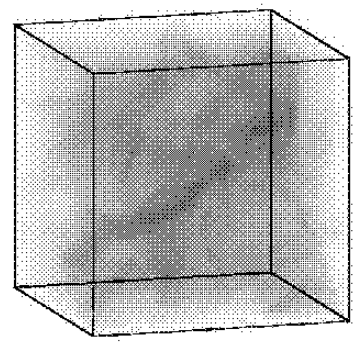

$$
t=0.0 \quad(k=1 \ldots 2)
$$$$
M_{*}=0.0 \%
$$

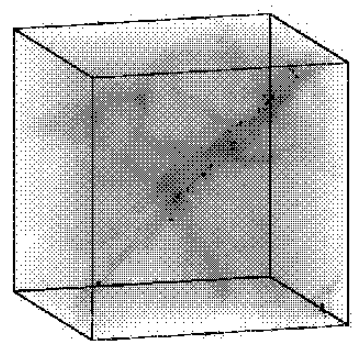

$$
\begin{aligned}
& t=1.3 \quad(k=1 \ldots 2) \\
& M_{*}=26.7 \%
\end{aligned}
$$

MODEL 4

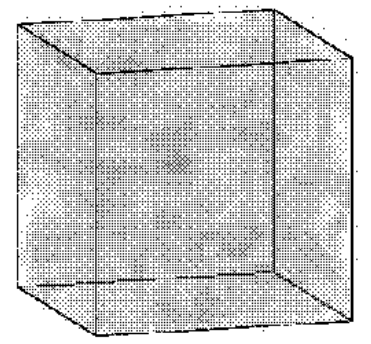

$t=0.0 \quad(k=3 \ldots 4)$ $M_{*}=0.0 \%$

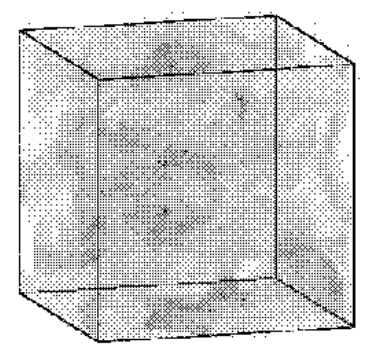

$$
t=1.8 \quad(k=3 \ldots 4)
$$$$
M_{*}=31.5 \%
$$

\section{MODEL 5}

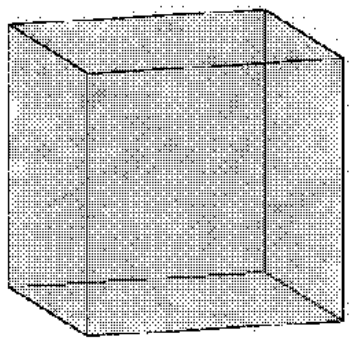

$$
t=0.0 \quad(k=7 \ldots 8)
$$$$
M_{*}=0.0 \%
$$

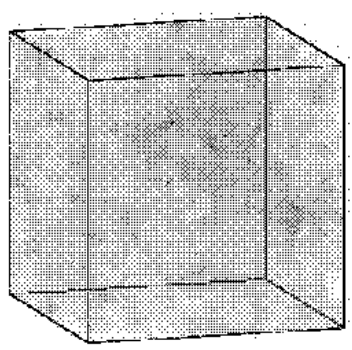

$$
t=4.0 \quad(k=7 \ldots 8)
$$

FIG. 1. - Comparison of the gas distribution in the five models at two different phases of the dynamical evolution, at $t=0$ indicating the initial density structure, just before gravity is 'switched on', and after the first cores have formed and accumulated roughly $M_{*} \approx 30 \%$ of the total mass. The high-density (protostellar) cores are indicated by black dots. Note the different time interval needed to reach the same dynamical stage. Time is normalized to the global free-fall timescale of the system, which is $\tau_{\mathrm{ff}}=10^{5} \mathrm{yr}$ for $T=11.4 \mathrm{~K}$ and $n\left(\mathrm{H}_{2}\right)=10^{5} \mathrm{~cm}^{-3}$. The cubes contain masses of $220\left\langle M_{\mathrm{J}}\right\rangle$ (models 1 and 2) and 120 $\left\langle M_{\mathrm{J}}\right\rangle$ (models 3 to 5), respectively, where the average thermal Jeans mass is $\left\langle M_{\mathrm{J}}\right\rangle=1 \mathrm{M}_{\odot}$ with the above scaling. The considered volumes are $(0.32 \mathrm{pc})^{3}$ and $(0.29 \mathrm{pc})^{3}$, respectively. Note, however, that the isothermal models are freely scalable as discussed in $\$ 2$ 


\section{MASS SPECTRA FROM TURBULENT FRAGMENTATION}

\subsection{The Mass Spectra}

Mass is the dominant parameter that determines stellar evolution. It is therefore important to investigate the relation between the mass of molecular clumps and protostellar cores, and the stars resulting from the collapse of the former. For the five models considered here, figure 2 plots the mass distribution of gas clumps (thin lines), of the subset of Jeans-critical clumps (thin lines, hetched distribution), and of collapsed cores (thick lines, hetched area). It depicts four different evolutionary phases, the initial distribution just when gravity is 'switched on' (at $t=0$, left column), and then after (turbulent) fragmentation has led to protostellar core formation, i.e. when the fraction $M_{*}$ of the total mass accumulated in dense cores has reached values of $M_{*} \approx 5 \%, M_{*} \approx 30 \%$, and $M_{*} \approx 60 \%$ (columns 2 to 4 , respectively). The clump mass spectra are obtained applying a clumpfinding algorithm similar to the one described by Williams et al. (1994), but working on all three spatial coordinates and adapted to make use of the SPH kernel smoothing procedure (for details see Appendix 1 in paper I). To guide your eye, two dotted lines indicate a slope $\nu=-1.5$ typical for the observed power-law clump mass spectrum $d N / d M=M^{\nu}$, as well as the Salpeter (1955) approximation $\nu=-2.33$ to the stellar IMF appropriate for intermediate and high masses (e.g. Scalo 1998, Kroupa 2001).

\subsection{Time Evolution of Clump Mass Spectra in the Interplay between Turbulence and Self-Gravity}

In the initial phase, i.e. before gravity is 'turned on' and local collapse begins to set in, the clump mass spectrum (thin line) is not well described by a single power law. The distribution has small width and falls off steeply at larger masses. Below masses $M \approx 0.3\left\langle M_{\mathrm{J}}\right\rangle$ the distribution becomes shallow, and strongly declines at and beyond the resolution limit (indicated by a vertical line). Clumps are on average considerably smaller than the mean Jeans mass in the system $\left\langle M_{\mathrm{J}}\right\rangle$. For masses $M>0.1\left\langle M_{\mathrm{J}}\right\rangle$ and for models 1 to 4 , this behavior resembles the spectrum of pre-stellar condensations found in $\rho$-Ophiuchus (Motte et al. 1998, Johnstone et al. 2000, see also Testi \& Sargent 1998 for Serpens). Recall that for densities of $n\left(\mathrm{H}_{2}\right)=10^{5} \mathrm{~cm}^{-3}$ and temperatures $T=11.4 \mathrm{~K}$, the mean Jeans mass in the system is $\left\langle M_{\mathrm{J}}\right\rangle=1 \mathrm{M}_{\odot}$.

In the later evolution the effects of gravitational attraction modify the distribution of clump masses. Clumps merge and grow bigger, and the mass spectrum extends towards larger masses. At the same time the number of cores which exceed the Jeans limit increases. Local collapse sets in and results in the formation of dense cores. This happens fastest and is most evident in model 1 which lacks turbulent support. The velocity field is entirely determined by gravitational contraction on all scales and at all times. The clump mass spectrum is very well fit by a single power law and exhibits a slope $\nu \approx-1.5$ as long as protostellar cores are forming and the overall gravitational potential is dominated by non-accreted gas.

The influence of gravity on the clump mass distribution is weaker where turbulence dominates over gravitational contraction on the global scales, i.e. in model 2 during the early stages and in models 3 to 5 during all phases. The more the turbulent energy dominates over gravity, the more the spectrum resembles the initial case of pure hydrodynamic turbulence. This is most extreme in model 5 of small-wavelength turbulence, where the short interval between shock passages prohibits ef- ficient clump merging and the build up of a large number of massive clumps. Only few clumps exceed the Jeans limit, become gravitationally unstable, and collapse to form cores. The bulk of the mass distribution remains unchanged by gravity and is never well fit by a single power law. The mass spectrum retains the initial shape with only few collapsed clumps added at the high-mass end.

When the scalelength of the dominant turbulent mode is increased, the density structure becomes more coherent and clump mergers are more frequent. The number of high-mass Jeans-unstable clumps increases, yielding a wider clump mass distribution which exhibits a power-law behavior for all masses larger than the resolution limit. In models 2 to 4 , the slope lies in the interval $-2.5<\nu \lesssim-2$. For individual models in figure 2, the slope $\nu$ increases with time as the statistical properties of the system become more and more influenced by clump merging and gravitational contraction onto high-density cores. When comparing similar evolutionary phases for different models, again the clump spectrum falls off less steeply if gravity dominates the evolution over larger spatial scales, i.e. $\nu$ decreases from model 1 to model 5 . When $M_{*} \approx 60 \% \nu \approx-1.5$ for model $1, \nu \lesssim-2$ for models 2 and 3, while for models 4 and 5 a power-law description is no longer sensible. In summary, the clump mass spectrum gets shallower when gravity becomes more important. This could explain the observed range of slopes $-1.9 \lesssim \nu \lesssim-1.3$ for the clumps mass spectra in different molecular cloud regions (e.g. Stutzki \& Güsten 1990, Williams et al. 1994, Heithausen et al. 1998, Kramer et al. 1998, Onishi et al. 1998). The importance of self-gravity for shaping the velocity and density structure may differ from cloud to cloud. In the case of strong gravity, their statistical properties furthermore depend sensitively on the viewing angle (e.g. Klessen 2000).

In the late evolutionary stages, similar behavior holds for the subset of Jeans-unstable clumps. For the three models of driven turbulence, the distribution of the gravitationally supercritical clumps (as indicated by hatched thin lines) is largest and widest for model 3 and decreases in width and size towards model 5. The clump spectra of models 2 and 3 are similar, indicating that the conditions for local collapse within the coherent shock-compressed regions which result from large-scale driving turbulence are comparable to turbulent decay. Within these coherent structures, turbulence is strongly reduced and stars formation is efficient. In the early stages of the evolution, however, the mass spectrum of Jeans-unstable clumps is not well described by a power law for all models, instead it is more compatible with a log-normal distribution. For the Gaussian model the peak is roughly at the average thermal Jeans mass $\left\langle M_{\mathrm{J}}\right\rangle$ and decreases towards smaller masses when including turbulence and decreasing the driving wavelength. The distribution of Jeans-unstable clumps in model 5 peaks roughly at $1 / 4\left\langle M_{\mathrm{J}}\right\rangle$. Thus small-scale turbulence produces clumps of on average smaller mass scale than does large-scale turbulence.

\subsection{Protostellar Mass Spectra from Turbulent Fragmentation}

Like the distribution of Jeans-unstable clumps, also the mass spectrum of dense protostellar cores (thick hatched line) resembles a log-normal in the model without turbulent support, and in the ones with turbulent decay or long-wavelength turbulent driving. A log-normal fit is obtained at times $M_{*} \approx 30 \%$ and $M_{*} \approx 60 \%$, and indicated by long-dashed lines in column 3 and 4. The corresponding mean values and widths are given in table 

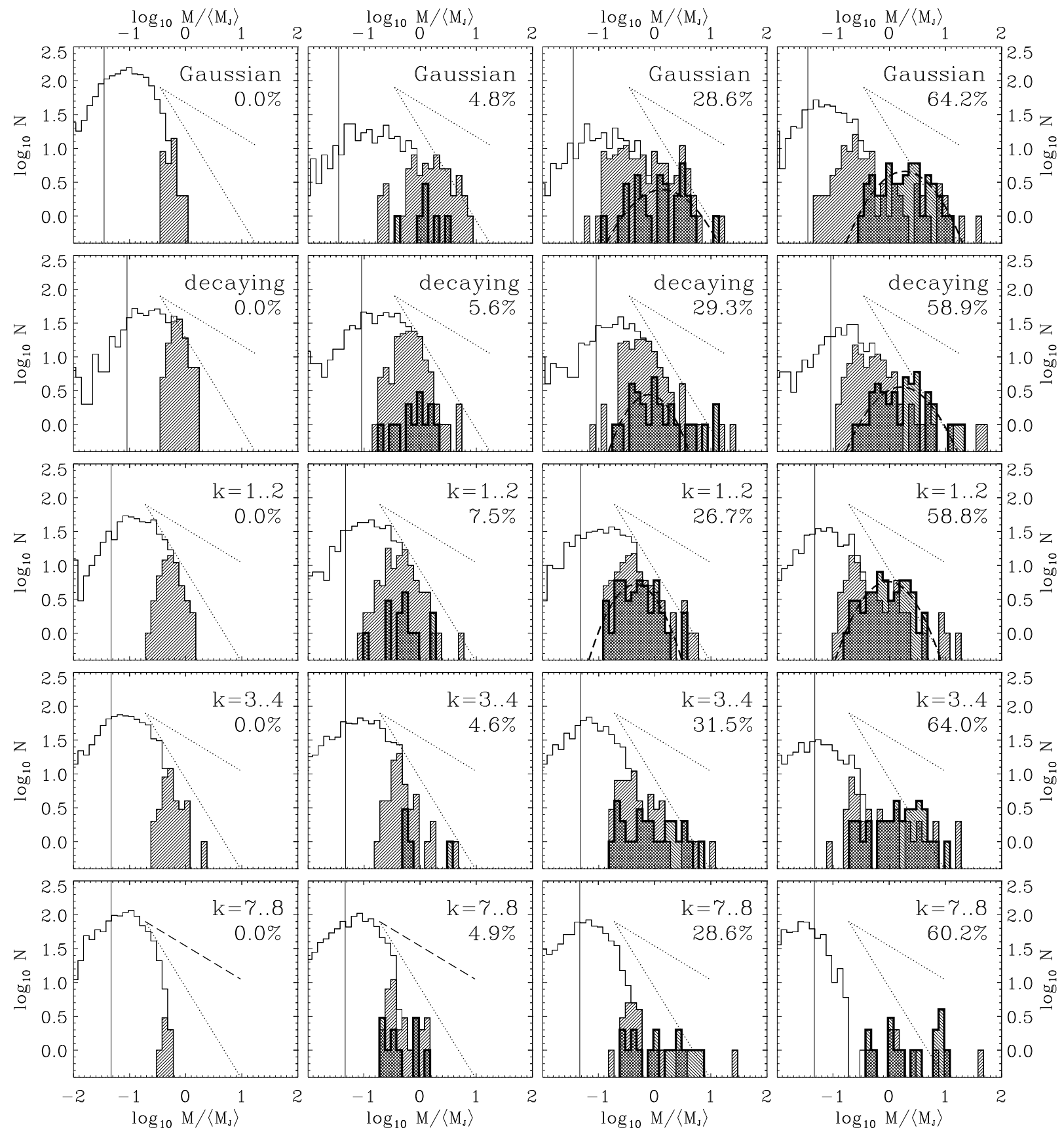

FIG. 2.- Mass spectra of gas clumps (thin lines), and of the subset of Jeans unstable clumps (thin lines, hatched distribution), and of dense collapsed cores (hatched thick-lined histograms). Masses are binned logarithmically and normalized to the average thermal Jeans mass $\left\langle M_{\mathbf{J}}\right\rangle$. The left column gives the initial state of the system, just when gravity is 'switched on', the second column shows the mass spectra when $M_{*} \approx 5 \%$ of the mass is accreted onto dense cores, the third column describes $M_{*} \approx 30 \%$, and the last one $M_{*} \approx 30 \%$. For comparison with power-law spectra $\left(d N / d M \propto M^{\nu}\right)$, a slope $\nu=-1.5$ typical for the observed clump mass distribution, and the Salpeter slope $\nu=-2.33$ for the IMF at intermediate and large masses, are indicated by the dotted lines in each plot. Note that with the adopted logarithmic mass binning these slopes appear shallower by +1 in the plot. The vertical line shows the SPH resolution limit. In columns 3 and 4 , the long dashed curve shows the best log-normal fit to the core mass spectrum. To compare the distribution of core masses with the stellar IMF, an efficiency factor of roughly $1 / 3$ to $1 / 2$ for the conversion of protostellar core material into single stars needs to be taken into account, as discussed in the text. For $T=11.4 \mathrm{~K}$ and $n\left(\mathrm{H}_{2}\right)=10^{5} \mathrm{~cm}^{-3}$, the average Jeans mass in the system is $\left\langle M_{\mathrm{J}}\right\rangle=1 \mathrm{M}_{\odot}$. Note, however, that the considered models can be scaled to different $\left\langle M_{\mathrm{J}}\right\rangle$ as well. 
2. As for the Jeans-critical cores, the peak is roughly at the $a v$ erage thermal Jeans mass $\left\langle M_{\mathrm{J}}\right\rangle$ of the system. The width of the distribution spans two orders of magnitude for the cluster size considered here, and is approximately the same for all five models. However, a log-normal fit is only appropriate for models 1 to 3 . The core mass spectrum for models 4 and 5 is too flat, and a fit is not attempted. Here the accretion histories of individual protostellar cores are not well correlated, i.e. gas clumps typically do not contain multiple protostellar cores. In this isolated mode of star formation, mutual interaction and competition for gas accretion are not important.

A log-normal shape of the mass distribution may be explained by invoking the central limit theorem (e.g. Larson, 1973, Zinnecker 1984, also Adams \& Fatuzzo 1996), as protostellar cores form and evolve through a sequence of highly stochastic events, resulting from the statistical nature of supersonic turbulence, stochastic clump merging, and/or competitive accretion within merged clumps containing multiple cores. The fact that the quality of the log-normal description becomes bad for the case of isolated star formation (model 5) indicates that clump merging and competitive accretion are dominant factors leading to a log-normal mass spectrum. Isolated star formation in supersonic turbulence exhibits a featureless flat mass spectrum. It appears that the collective effects of gravitational collapse and the synchronization of individual accretion histories (i.e. the mutual interaction of protostellar cores and their competition for accretion from a common gas reservoir) are necessary to obtain mass spectra with features similar to the IMF $(\$ 4.4)$. The fact that the distribution peaks roughly at the average Jeans mass, despite strong variations of the local Jeans mass for individual clumps which span a wide range of densities, indicates that the system retains in a statistical sense knowledge of its mean properties, even in the case of supersonic turbulence. The same behavior is seen in paper's I and II for a wide varyity of Gaussian initial conditions. It is the average thermal Jeans mass that introduces a scale into the mass function (e.g. Larson 1998, Elmegreen 1999).

\subsection{Comparison with the Stellar Mass Function}

For the direct comparison between the core mass spectrum and the IMF one needs to adopt a physical scaling for the numerical model $(\$ 2)$. This is done here by taking the average thermal Jeans mass to be $\left\langle M_{\mathrm{J}}\right\rangle=1 \mathrm{M}_{\odot}$. Furthermore, one needs to estimate which fraction of a typical protostellar core in the model will accrete onto the star(s) in its interior, and which fraction may be expelled during the main accretion phase by protostellar outflows, or be removed by tidal effects in a clustered environment. For isolated cores forming single stars, the mass loss due to radiation or outflows is expected to be small and most of the core material will indeed end up in stars (Wuchterl \& Tscharnuter 2000). In this case the core mass spectrum can be compared directly to the single-star IMF. If cores contain a large amount of angular momentum, they are likely to form binary stars. Indeed a initial binary fraction of almost $100 \%$ is consistent with observations of star clusters (Kroupa 1995). Assuming a more or less uniform distributon of mass ratios (Duquennoy \& Mayor 1991) leads to a shift of a factor of two in the characteristic mass of the distribution compared to the single-star IMF. If the number of triple systems is high, this shift could be even larger. To some degree this effect can be taken into account by comparing the core mass spectrum with IMF estimates that do not contain binary corrections (see Kroupa 2001 for a further discussion). In addition, a fraction of the accreting matter may settle into a protobinary disk and may not accrete onto the stars due to angular momentum conservation (e.g. Bate 2000). In a cluster environment, this disk may be truncated and leak out matter due to tidal interactions (e.g. Clarke, Bonnell, \& Hillenbrand 2000). These effects depend strongly on the stellar density of the cluster and its dynamical evolution. Altogether, the peak in the mass spectrum of protostellar cores is expected to exceed the characteristic mass in the single star IMF by a factor of 2 to 3 (i.e. is shifted by $\left.0.3 \lesssim \Delta \log _{10} M \lesssim 0.5\right)$.

Additional uncertainty stems from the possible formation of $\mathrm{O}$ or B stars in the stellar cluster. These would trigger the complete gas removal due to ionizing radiation, therefore limiting the core formation efficiency $M_{*}$. As it is not known in advance whether at all and when high mass stars form during the protostellar cluster evolution, the current models should be considered with caution at very late phases $M_{*}>70 \%$.

If one assumes a close correspondence between core masses and stellar masses, then for models 1 to 3 the core mass distribution compares well with the observed IMF at low to intermediate masses. The width of the distribution falls right in between the log-normal IMF estimates by Miller \& Scalo (1979) on one side and by Kroupa et al. (1990) and Scalo (1986) on the other. The same holds when using the more common multiple power-law description of the IMF (Scalo 1998 or Kroupa 2001, see e.g. his figure 14). Also the characteristic masses in the distribution become comparable if one adopts values of few $\times 0.1$ for the accretion efficiency from cores to the central stars taking into account unresolved binaries and possible tidal truncation of protobinary disks in a dense cluster environment as mentioned before. However, more work needs to be done to obtain better estimates for this efficiency factor. This requires combining the simulations described here with detailed dynamical models of pre-main sequence evolution, and extending the current scheme to resolving larger density contrasts and larger clusters sizes to be able to study in more detail the influence of cluster environment on the dynamical evolution of accretion disks.

The protostellar clusters discussed here contain between 50 and 100 cores. This allows for comparison with the IMF only around the characteristic mass scale, i.e. at low to intermediate masses. The numbers are too small to study the very lowand high-mass end of the distribution. The same holds for the mass spectra discussed in paper I and II. For high-mass stars $\left(M \gtrsim 5 \mathrm{M}_{\odot}\right)$ the $\log$-normal and the multiple power-law descriptions of the IMF begin to differ significantly. The lognormal models predict too few high-mass stars, and the same may also be true at the very low-mass end of the IMF, in the brown dwarf regime (especially when taking binary corrections into account). Because of insufficient statistics at the extreme ends of the distribution, the current set of simulations cannot be used to distinguish between log-normal and power-law IMF models. The log-normal fit at low to intermediate mass in figure 2 is therefore mainly attempted for the sake of simplicity, because only two parameters, the peak value and the width, are sufficient to characterize the distribution. Both can be conveniently compared with the corresponding fit parameters for the stellar mass function in the considered mass range.

The comparison reveals a striking contrast between the models of turbulent decay or large-scale driving on the one side, and models of short- to intermediate-wavelength turbulence on the other. Models 1 to 3 lead to protostellar mass spectra that agree well with the observations, whereas the mass spectra derived 
TABLE 2

Properties OF LOG-NORMAL FIT

\begin{tabular}{|c|c|c|c|c|c|c|c|c|c|c|}
\hline Parameter & \multicolumn{2}{|c|}{$\begin{array}{c}\text { model } 1 \\
\sim 30 \% \sim 60 \%\end{array}$} & \multicolumn{2}{|c|}{$\begin{array}{c}\text { model } 2 \\
\sim 30 \% \sim 60 \%\end{array}$} & \multicolumn{2}{|c|}{$\begin{array}{c}\text { model } 3 \\
\sim 30 \% \sim 60 \%\end{array}$} & \multicolumn{2}{|c|}{$\begin{array}{c}\text { model } 4 \\
\sim 30 \% \sim 60 \%\end{array}$} & \multicolumn{2}{|c|}{$\begin{array}{c}\text { model } 5 \\
\sim 30 \% \sim 60 \%\end{array}$} \\
\hline $\log _{10} \mu$ (peak) & 0.13 & 0.26 & -0.10 & 0.21 & -0.34 & -0.03 & - & - & - & - \\
\hline $\log _{10} \sigma$ (width) & 0.52 & 0.47 & 0.36 & 0.47 & 0.36 & 0.40 & - & - & - & - \\
\hline
\end{tabular}

NOTE.- The log-normal fits are obtained for models 1 to 3 only for two different evolutionary stages, when $M_{*} \approx 30 \%$ and $M_{*} \approx 60 \%$. The applied functional form is $d N / d \log _{10} M \propto \exp \left[-0.5\left(\log _{10} M-\log _{10} \mu\right)^{2} /\left(\log _{10} \sigma\right)^{2}\right]$, with mass $M$, mean $\mu$ and width $\sigma$ scaled to the average Jeans mass in the system $\left\langle M_{\mathrm{J}}\right\rangle$. No fit has been obtained for models 4 and 5 , the core mass spectrum is too flat and featureless. When scaling the current models to physical units, the width of the core mass distributon lies between the IMF estimates by Miller \& Scalo (1979) and by Scalo (1986) and Kroupa et al. (1990), who derive $\log _{10} \sigma=0.67$ (their estimate with constant star formation rate over $12 \times 10^{9}$ years) and $\log _{10} \sigma=0.38$ (their model MS), respectively. Recall that $\left\langle M_{\mathrm{J}}\right\rangle=1 \mathrm{M}_{\odot}$ for $n\left(\mathrm{H}_{2}\right)=10^{5} \mathrm{~cm}^{-3}$ and $T=11.4 \mathrm{~K}$. As the number of protostars in the simulated cluster is limited to $50-100$, the comparison with the stellar IMF applies to low to intermediate-mass stars only. The statistics is not good enough for an investigation of the very low-mass and the high-mass end of the IMF where the log-normal parametrization fails.

from models 4 and 5 compare only very poorly with the stellar IMF. They are too flat or equivalently too wide. As small- to intermediate-scale turbulence describe an isolated mode of star formation, this finding is consistent with the hypothesis that most stars form in aggregates or clusters (e.g. Adams \& Myers 2001). To further constrain the numerical models discussed here, it will be necessary to compute the dynamical evolution of star-forming regions with 1000 protostellar cores or more. Besides the width and characteristic mass of the core distribution, also the detailed slope at very low and very high masses and the apparent symmetry around the peak can then be included into the analysis.

Finally, it needs to be noted that the current findings raise doubts about attempts to explain the stellar IMF from the turbulence-induced clump mass spectrum only (e.g. Elmegreen 1993, Padoan 1995, Padoan et al. 2001, Padoan \& Nordlund 2001). Quite typically for star forming turbulence, the collapse timescale of shock-compressed gas clumps often is comparable to their lifetime (molecular cloud clumps appear to be very transient, e.g. Bergin et al. 1997). This not only has important consequences for the overall star formation efficiency in turbulent clouds (Klessen et al. 2000), but more so for the collapse behavior of individual Jeans-unstable shock-generated gas clumps. While collapsing to form or feed protostars, clumps may loose or gain matter from interaction with the ambient turbulent flow. In a dense cluster environment, collapsing clumps may merge to form larger clumps containing multiple protostellar cores, which subsequently compete with each other for accretion form the common gas environment (Bonnell 1997, paper's I and II). The resulting distribution of clump masses in star forming regions strongly evolves in time (figure 2). In dense clusters, furthermore, close encounters between accreting protostars may become important leading to the expulsion of protostars from the gas rich environment (as illustrated in figure 11 of paper I). This terminates mass growth and if occuring frequently enough modifies the resulting IMF. The mass accretion rates onto individual protostars are highly stochastic and strongly depend on the cluster environment (paper III). For all this reasons, it is not possible to infer a one-to-one relation between the clump masses resulting from turbulent molecular cloud fragmentation and the stellar IMF. Given our limited understanding of interstellar turbulence and protostellar mass growth processes in dense clusters, the current investigation (which attempts to include some of the above processes) leads to the conclusion that - although tempting in some cases - it is not appropriate to take a snapshot of the turbulent clump mass spectrum as describing the IMF.

\section{SUMMARY}

Stars form from turbulent fragmentation of molecular cloud material. It is the relation between turbulent fragmentation, (localized) gravitational collapse and star formation which is the focus of this paper. As mass is the most important stellar parameter, particular interest lies in the mass spectra of gas clumps, of the subset of gravitationally unstable gas clumps, and of protostellar cores, the latter one being the direct progenitors of stars. For this purpose five numerical models of the evolution of selfgravitating isothermal molecular gas have been analyzed, spanning the parameter range relevant for molecular cloud dynamics. In model 1 turbulent support is not included and gravity is the dominant force shaping the velocity and density structure. In model 2 , initially supersonic compressible turbulence is allowed to decay freely, and in models 3 to 5 supersonic turbulence is continuously replenished on large, intermediate and small scales, respectively, such that gravitational attraction is compensated on global scales. In these models gravity is considered only after turbulent equilibrium is established.

It has been shown in a previous study (papers I and II) that molecular cloud regions without turbulent support form dense clusters of stars, regardless of the initial density structure, within roughly one global free-fall timescale. When gravitational contraction has sufficient time to act, the clump mass spectrum is well approximated by a power law $d N / d M \propto$ $M^{\nu}$. The mass distribution of protostellar cores, however, is better described by a log-normal with properties similar to the observed IMF of multiple stellar systems for low and intermediate-mass stars.

This analysis is extended here by more realistically consid- 
ering molecular cloud regions where turbulence is allowed to decay and where it is continuously driven. Decaying turbulence leads to clustered star formation much like in the case of pure gravitational contraction. Supersonic turbulence, even if it is strong enough to compensate gravity on large scales, will provoke local collapse in shock compressed regions. As efficiency and timescale of star formation depend sensitively on the strength and the spatial scale of energy input into the system, large-scale turbulence leads to clustered star formation on short timescales, whereas for small-scale turbulence stars form in isolation and with low efficiency.

This is reflected in the overall clump mass spectrum. The clump mass spectrum of pure hydrodynamic turbulence is not well described by a single power law, its width is too small compared to the observed data and it is too steep at the high-mass end. This changes, when gravity is taken into account. Clumps merging and accumulation of matter through local collapse, lead to a clump mass spectrum that extends to larger masses and that exhibits power-law behavior. The more strongly the overall dynamical evolution is influenced by gravity and synchonized, coherent collapse behavior, the flatter the power spectrum becomes. In the extreme case of pure gravitational contraction the clump mass distribution exhibits a slope $\nu \approx-1.5$. For the case of turbulence decay and large-scale injected turbulence the slope is $\nu \lesssim-2$ during the intermediate phases of the dynamical evolution. In the case of small-scale turbulence, the infuence of gravity is weak and the clump mass distribution remains steep, close to the spectrum of purely hydrodynamic turbulence (i.e. before gravity was 'switched on' in the model). The dependence of the slope of the clump mass spectrum on the relative importance of gravity may explain the range of observed power-law indices in different molecular clouds regions, as one expects the ratio between self-gravity and turbulent kinetic energy to vary from cloud to cloud.

Molecular cloud properties that result in clustered star forma- tion lead a stellar mass spectrum that is well fit by a log-normal at low and intermediate masses. The distribution exhibits a maximum close to the average Jeans mass in the system. This indicates that the system somehow retains 'knowledge' of its mean properties, even in the case of supersonic compressible turbulence. The mean thermal Jeans mass in a cloud indeed introduces a characteristic mass scale to clustered star formation. For regions where turbulence is decaying or driven on large scales only, it appears that the collective effects of gravitational collapse and the correlation between individual accretion histories are necessary to obtain mass spectra with features similar to the IMF. Isolated star formation, on the contrary, as implied by turbulence that is driven on small scales, yields a featureless flat spectrum. This is in agreement with the hypothesis, that most stars form in aggregates and clusters.

Shock-generated clumps in interstellar turbulence are highly transient. Their average lifetime in the turbulent flow is of the same order of their collapse timescale. In a dense cluster environment, furthermore competitive accretion and mutual protostellar interactions are important effects. The current investigation shows that it is therefore not possible to infer a one-to-one relation between turbulent clump mass spectra and the stellar IMF.

I thank Peter Bodenheimer, Andreas Burkert, Fabian Heitsch, Pavel Kroupa, Doug Lin, Mordecai-Mark Mac Low, and Günther Wuchterl for many stimulating discussions on star formation and the IMF and/or fruitful collaboration. I furthermore appreciate the comments and suggestions of the referee John Scalo. They helped to clarify the arguments presented here. I acknowledge financial support by a Otto-HahnStipendium from the Max-Planck-Gesellschaft and partial support through a NASA astrophysics theory program at the joint Center for Star Formation Studies at NASA-Ames Research Center, UC Berkeley, and UC Santa Cruz.

\section{REFERENCES}

Adams, F. C., Fatuzzo, M., 1996, ApJ, 464, 256

Adams, F. C., Myers, P. C., 2001, ApJ, in press astro-ph/0102039)

Ballesteros-Paredes, J., Vázquez-Semadeni, E., Scalo, J., 1999, ApJ, 515, 286

Bate, M. R., 2000, MNRAS, 314, 33

Bate, M. R., Bonnell, I. A, Price, N. M., 1995, MNRAS, 277, 362

Benz, W., 1990, in The Numerical Modeling of Nonlinear Stellar Pulsations, ed. J. R. Buchler (Dordrecht: Kluwer), 269

Bonnell, I. A., Bate, M. R., Clarke, C. J., \& Pringle, J. E. 1997, MNRAS, 285, 201

Clarke, C. J., Bonnell, I. A., Hillenbrand, L. A., 2000, in Protostars and Planets, eds. V. Mannings, A. P. Boss, \& S. S. Russell, University of Arizona Press, Tucson, p. 151

Duquennoy, A., Mayor, M., 1991, A\&A, 248, 485

Ebisuzaki, T., Makino, J., Fukushige, T., Taiji, M., Sugimoto, D., Ito, T., Okumura, S. K., 1993, PASJ, 45, 269

Elmegreen, B. G. 1993, ApJ, 419, L29

Elmegreen, B. G. 1993, MNRAS, 311, L5

Bergin, E. A., Goldsmith, P. F., Snell, R. L., Langer, W. D., 1997, ApJ, 428, 285

Heithausen, A., Bensch, F., Stutzki, J., Falgarone, E., Panis, J. F., 1998, A\&A, 331, L65

Heitsch, F., Mac Low, M.-M., \& Klessen, R. S. 2001, ApJ, 547, 280

Henriksen, R., André, P., Bontemps, S., 1997, A\&A, 323, 549

Johnstone, D., Wilson, C. D., Moriarty-Schieven, G., Joncas, G., Smith, G., Gregersen, E., Fich, M., 2000, 545, 327

Klessen, R. S., 1997, MNRAS, 292, 11

Klessen, R. S., 2000, ApJ, 535, 869

Klessen, R. S., 2001, ApJ, 550, L77 (paper III)

Klessen, R. S., Burkert, A., 2000, ApJS, 128, 287 (paper I)

Klessen, R. S., Burkert, A., 2001, ApJ, 549, 386 (paper II)

Klessen, R. S., Burkert, A., \& Bate, M. R., 1998, ApJ, 501, L205

Klessen, R. S., Heitsch, F., Mac Low, M.-M., 2000, ApJ, 535, 887

Kramer, C., Stutzki, J., Rohrig, R., Corneliussen, U., 1998, A\&A, 329, 249

Kroupa, P., 1995, MNRAS, 277, 1491

Kroupa, P., 2001, MNRAS, 322, 231
Kroupa, P., Tout, C. A., Gilmore, G., 1990, MNRAS, 244, 76

Lada, E., 1992, ApJ, 393, L25

Larson, R. B., 1973, MNRAS, 161, 133

Larson, R. B., 1998, MNRAS, 301, 569

Mac Low, M.-M., 1999, ApJ, 524, 169

Mac Low, M.-M., Klessen, R. S., Burkert, A., Smith, M. D., 1998, Phys. Rev. Lett., 80, 2754

Miller, G. E., Scalo, J. M., 1997, ApJS, 41, 513

Mizuno, A., Onishi, T., Yonekura, Y., Nagahama, T., Ogawa, H., Fukui, Y. 1995, ApJ, 445, L161

Motte, F., André, P., Neri, R., 1998, A\&A, 336, 150

Myers, P. C., Evans, N. J. , Ohashi, N., 2000, in Protostars and Planets, eds. V. Mannings, A. P. Boss, \& S. S. Russell, University of Arizona Press, Tucson, p. 217

Onishi, T. and Mizuno, A. and Kawamura, A. and Ogawa, H and Fukui, Y., 1996, ApJ, 465, 815

Ossenkopf, V., Mac Low, M.-M., 2001, A\&A, submitted (astro-ph/0012247)

Padoan, P., 1995, MNRAS, 277, 337

Padoan, P., Nordlund, A., 1999, ApJ, 526, 279

Padoan, P., Nordlund, A., 2001, ApJ, submitted astro-ph/0011465

Padpan, P. Juvela, M., Goodman, A. A., Nordlund, A., 2001, ApJ, submitted (astro-ph/0011122)

Salpeter, E. E., 1955, ApJ, 121, 161

Scalo, J., 1986, Fund. of Cos. Phys., 11, 1

Scalo, J., 1998, in The Stellar Initial Mass Function (38th Herstmonceux Conference), eds. G. Gilmore \& D. Howell, ASP Conference Series, Vol. 142, p. 201

Scalo, J., Vázquez-Semadeni, E., Chappell, D., Passot, T., 1998, ApJ, 504, 835

Shu, F. H. 1977, ApJ, 214, 488

Shu, F. H., Adams, F. C., Lizano, S., 1987, ARA\&A, 25, 23

Spaans, M., Silk, J., 2000, ApJ, 538, 115

Steinmetz, M., 1996, MNRAS, 278, 1005

Stutzki, J., Güsten, R., 1990, ApJ, 256, 513

Stone, J. M., Ostriker, E. C., Gammie, C. F., 1998, ApJ, 508, L99 
Sugimoto, D., Chikada, Y., Makino, J., Ito, T., Ebisuzaki, T., Umemura, M., 1990, Nature, 345, 33

Williams, J. P., Blitz, L., McKee, C. F., 2000, in Protostars and Planets IV, eds. V. Mannings, A. P. Boss, \& S. S. Russell, University of Arizona Press, Tucson, p. 97
Williams, J. P., De Geus, E. J., Blitz, L., 1994, ApJ, 428, 693

Wuchterl, G., Tscharnuter, W. M., 2000, A\&A, submitted

Testi, L., Sargent, A. I., 1998, ApJ, 506, L91

Testi, L., Palla, F., Natta, A., 1998, A\&A, 342, 515

Zinnecker, H., 1984, MNRAS, 210, 43 